\title{
Adaptive Load Balancing Strategy Based on LVS
}

\author{
Chen $\mathrm{FU}^{1, *}$, Li-Jun ZHANG ${ }^{2}$ \\ ${ }^{1}$ roomF217, new main building, Beihang University, No.37, xueyuanroad, HaiDian District, BeiJing, China \\ ${ }^{2}$ room G807, new main building, Beihang University, No.37, xueyuanroad, HaiDian District, BeiJing, China \\ ljzhang@buaa.edu.cn \\ *Corresponding author: fuchen319@163.com
}

\begin{abstract}
Linux Virtual Server (LVS) is a load balancing server that deployed on a cluster of real servers and the load balancer running on the Linux operating system. In the scheduling module, we designed an adaptive load balancing strategy. Firstly, we introduce the traffic state prediction. The algorithm we choose is Radial Basis Function (RBF) neural network. Secondly, we update weights according to the real-time usage of CPU, memory and hard disk. Then we conduct a series of experiments and the adaptive strategies have a better performance on load balancing.
\end{abstract}

\section{Introduction}

With the continuous development of network technology, the Page View of users and the load of servers are increasing exponentially. How to provide high quality and uninterrupted network services to users becomes a difficult problem for the Internet Service Provider(ISP) to solve. The use of a single server architecture is difficult to meet this demand, so the model of server farm[1] makes the problem solved. Server clustering techniques is a group of independent, high-speed Internet through a computer interconnected into a whole, and through a single system model to be managed. When the users interact with the cluster, the cluster is just like a separate server. As a kind of Server clustering techniques, the Linux LVS has been widely concerned and applied. It is a highly scalable and available server that deployed on a cluster[2] of real servers and the load balancer running on the Linux operating system. The architecture of the real servers is transparent to internet users, and the internet users interact as if it was a single high-performance server. The LVS can be used to build highly scalable and highly available network services as an advanced load balancing solution.

This paper includes three sections: first, we introduce the LVS model that how to guarantee the scalability and availability[3] with a cluster of real servers. Second, we introduce the new adaptive load balancing strategy. We designed a new scheduling algorithms base on the traffic state prediction and the changing weights of each real servers. We used RBF neural network[4] to predict the future network traffic. The weights is calculated according to the real-time usage of CPU, memory and hard disk at a certain time interval. Finally, we conduct a series of experiments. That compare the performance of the new adaptive load balancing strategy with other simple strategies and give a comprehensive evaluation. Then we discuss the future work that how to improve the performance.

\section{Server Load Balance and LVS}

\subsection{Server Load Balance and LVS Introduction}

Virtual server is a scalable and available server built on a cluster of loosely coupled independent real servers[5]. The architecture of the cluster is fully transparent to request clients outside the cluster. Clients interact with the cluster as if it was a single high-performance and highly available web server. The request clients are not affected by the interaction with that cluster and not need modification[6]. The architecture of a virtual server is showed in Fig.1. 


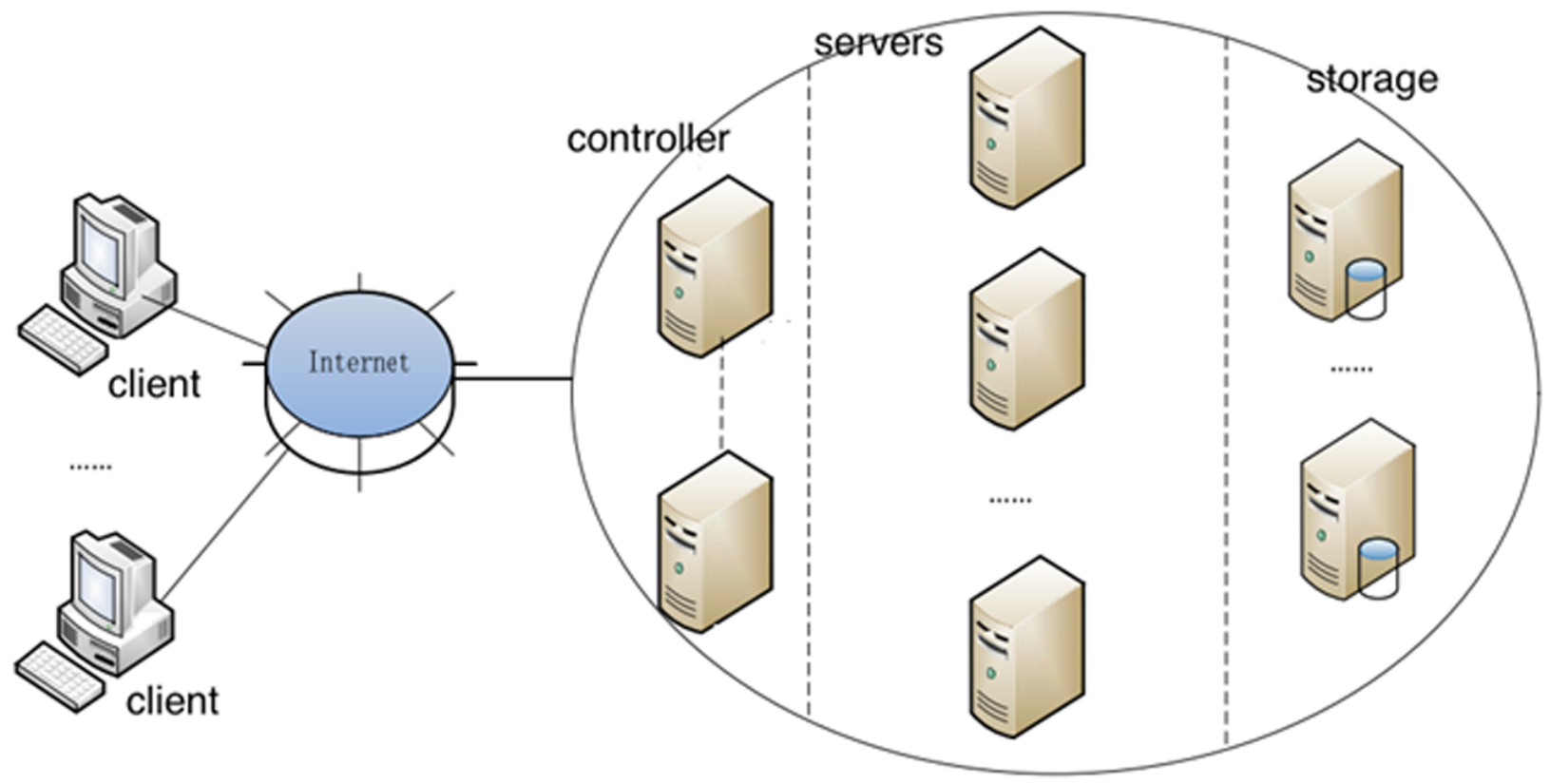

Figure 1. The architecture of a virtual server

The real servers are interconnected by the high-speed LAN or by the geographically dispersed WAN. The load balancer is the front-end of the real servers. It schedules requests packets to the real servers and make parallel services[7] of the cluster to appear as a virtual service on a virtual IP address. Scalability is achieved by transparently adding or removing a real server in the cluster. High availability is provided by detecting node and reconfiguring the cluster appropriately.

\subsection{Load Balance Based on LVS}

The traditional network service providers always use sever farm and load balancer to improve the availability and scalability of network service[8]. That always has two situations for traditional load balance on servers, one is using the same functional servers to provide the unified service; the other one is using different type servers to provide different services. For the first type, every internet user can obtain the service from any one real server just as the system only has one real server. While in the second one, each server provide same or different type of service, then we must distribute the requests data packets to different servers according to the packets content. In this paper, we only focus on the first one.

Round-robin scheduling algorithm, just as its word meaning, directs the request packets to the real servers in a round-robin manner. It treats all real servers as equals regardless of number of connections or response time.

The weighted round-robin scheduling can treat the real servers of different processing capacities. Each server will be assigned an integer that indicates the processing capacity. The default number is 1[9]. However, it may lead to dynamic load imbalance if the load of requests vary highly. In this paper, we calculate the weight according to the real-time usage of CPU, memory and hard disk at a certain time interval.

\section{Traffic State Prediction with RBF and Load Balance Process}

In this paper, we present a new internet load balancing scheduling algorithm that using the past number of data packets to predict the future number in a certain time, then we count the probability of each real server with the predicted number. With the data collecting module of LVS, we read the collected data at regular intervals and compute the future data packets number, then send the number to the RBF Scheduling algorithm module to distribute the data packets. The prediction algorithms used here is designed base on the RBF neural network.

\subsection{The RBF Neural Network}

The structure of RBF is showed as Fig.2. It is three layers artificial neural network, which are input layer, hidden layer and output layer. 


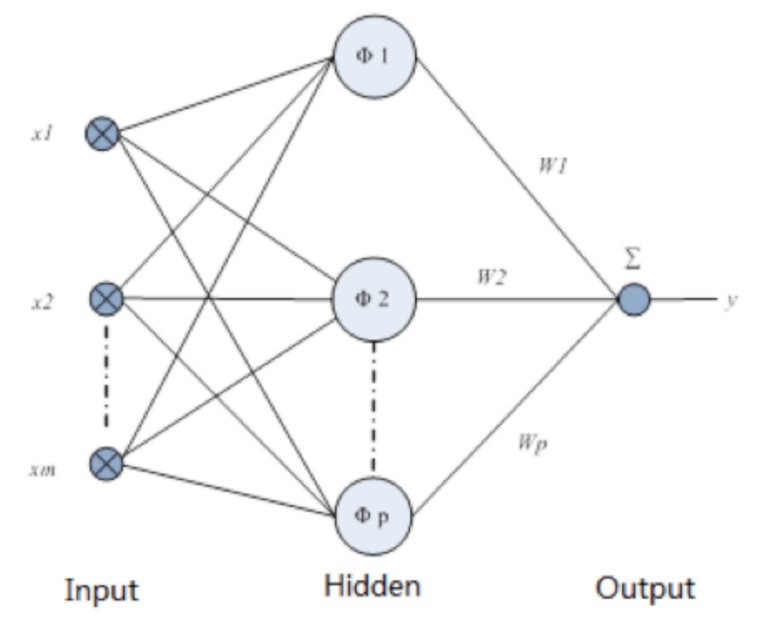

Figure 2. The RBF neural network

$x(k)=\left[x_{1}, x_{2}, x_{3}, \ldots, x_{m}\right]$ is the input layer and $\mathrm{m}$ is the number of input units. $\Phi=\left[\phi_{1}, \phi_{2}, \ldots, \phi_{p}\right]$ is the hidden layer and $\mathrm{p}$ is the number of hidden layer units. The ouput layer is Y. The following Eq.1 shows the output if RBF.

$$
\mathrm{F}(\mathrm{x})=\sum_{p}^{P} W_{p} \Phi_{p}\left\|X-X_{p}\right\|
$$

We used Gaussian function as the RBF function in the hidden layer, which is showed by the following Eq.2.

$$
r_{j}=\exp \left\lfloor-\frac{\left\|x-c_{j}\right\|^{2}}{2 \delta_{j}^{2}}\right\rfloor \cdot j=1,2, \ldots, p
$$

The output layer is the sum of the hidden layer, $Y=F(x)$.

We use the gradient descent method[10] to solve RBF, which can simplify the solving process. The first step is training all the parameters of the network with supervised learning algorithm. The parameters include the center of radial basis function $c_{j}$, variance $\delta$ and the weight between hidden layer and output layer. The center $c_{j}$ can be computed by k-means clustering function from the input samples. And in order to avoid the radial basis function too sharp or too flat, the variance $\delta$ is counted by Eq.3. $d_{\max }$ is the max distance between centers and $M$ is the number of centers.

$$
\delta=\frac{d_{\max }}{\sqrt{2 M}}
$$

Secondly, we use the gradient descent method to train the RBF neural network. The deviation from real output to desired output is the cost function, just as Eq.4 shows.

$$
\mathrm{E}=\frac{1}{2}(y(x)-d)^{2}
$$

In every training, the parameters are decreased in gradient-decent by the speed as Eq.5.

$$
\Delta w_{j}=-\eta \frac{\partial \mathrm{E}}{\partial w_{j}}=\eta \sum_{i=1}^{P} e_{j} G\left(\left\|X_{i}-c_{j}\right\|\right)
$$

The training process is doing until the value of the cost function meets the accuracy we need. So the algorithm can predict the traffic arrived in the next interval with RBF network and computes the probability of each real server. The next time, an internet load report packet arrived, the RBF will be training again.

\subsection{The Process of Server Load Balance Based on LVS}

The whole process of the server load balance presented in this paper is:

1 When the host send packets to the virtual IP, the load balancer will reveive the packets. The data statistics module will count the number of packets, and then send the packets to the scheduling module one by one. Different distribute algorithm can be selected for scheduling module, such as round-robin and weighted round-robin. We designed random, past load pressure and status predication scheduling algorithm in this module.

2 For the predication scheduling algorithm, a calculation script is executed at regular intervals. It will read the statistics of the data statistics module, and count the future traffic by the past data statistics. Finally, it will update the prediction data for the status predication scheduling algorithm.

3 A weight getting script is also executed at regular intervals. It send request information to every real server. The weight calculating scripts on real server will read the usage of CPU, memory and hard disk, then calculate the weight of the current state and send the result back. Finally, the script on the load balancer will update the weights of real servers.

4 The real server receive the request packets distributed by the load balancer. After complete the processing of the request, it send the result to the request host directly. 


\section{Experiments and Results}

The experiments conducted here are under LVS load balancing architecture. The hosts and servers is created under the Vmware Workstation. We focus on the load pressure of servers, and also test the link delay and packet loss rate between the host and the real server. Iperf is the test tool to send data packets, and we created
200 virtual machines as hosts and 8 real machine as servers here. The server IP is 192.168.50.160. The 200 hosts send the packets to the IP at a random interval. The average link delay, packets lost rate and the square deviation of the data packets number will be counted as the key test object.

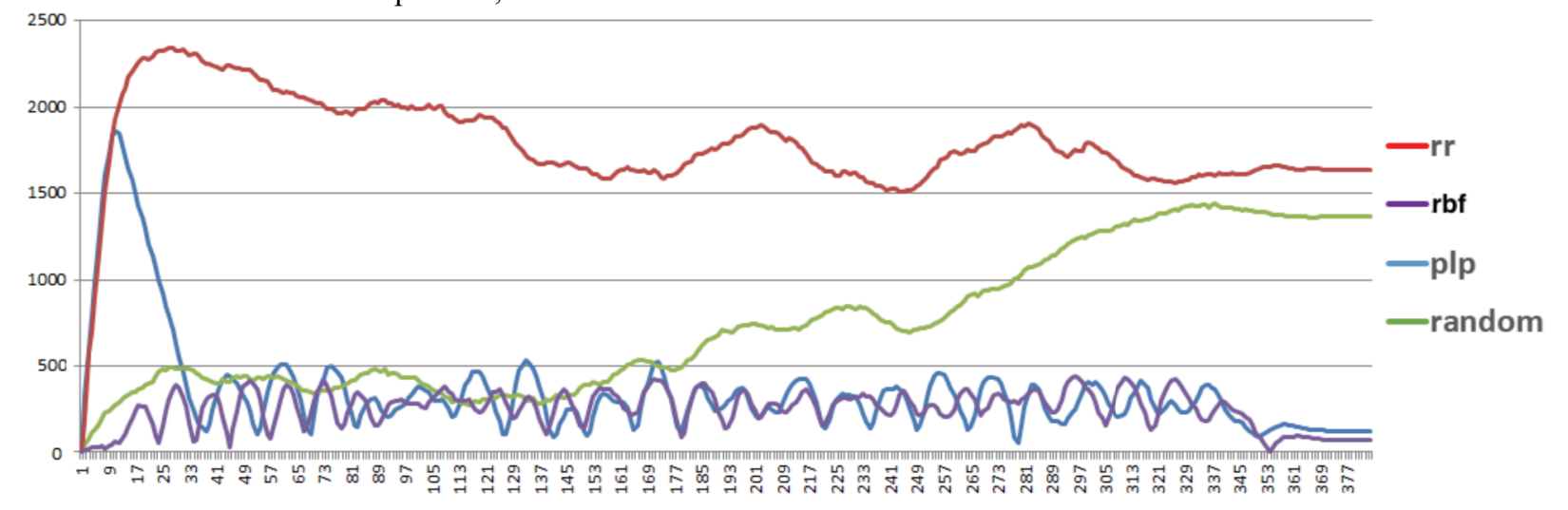

Figure 3. The square deviation of packets

The square deviation of data packets number accepted by all the real servers is showing as Fig.3. We can clearly see that the prediction method based on RBF and the simple past load pressure performed much better than the random and round-robin and RBF curve is more gentle, so its performance is the best stability.

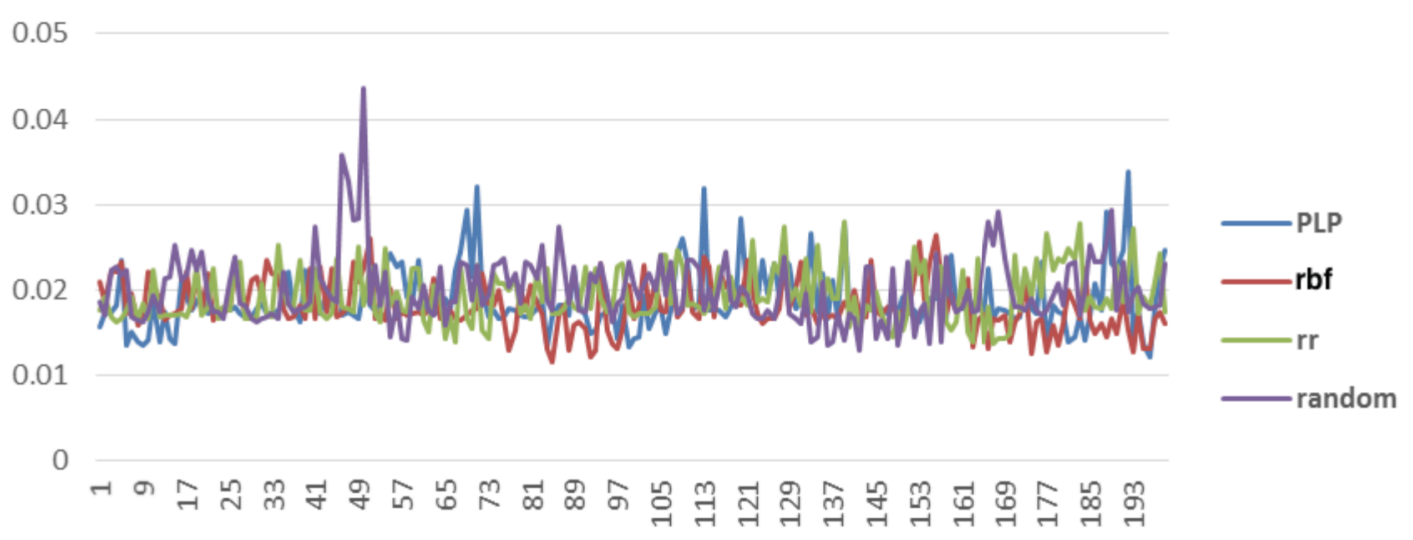

Figure 4. The link delay of packets 


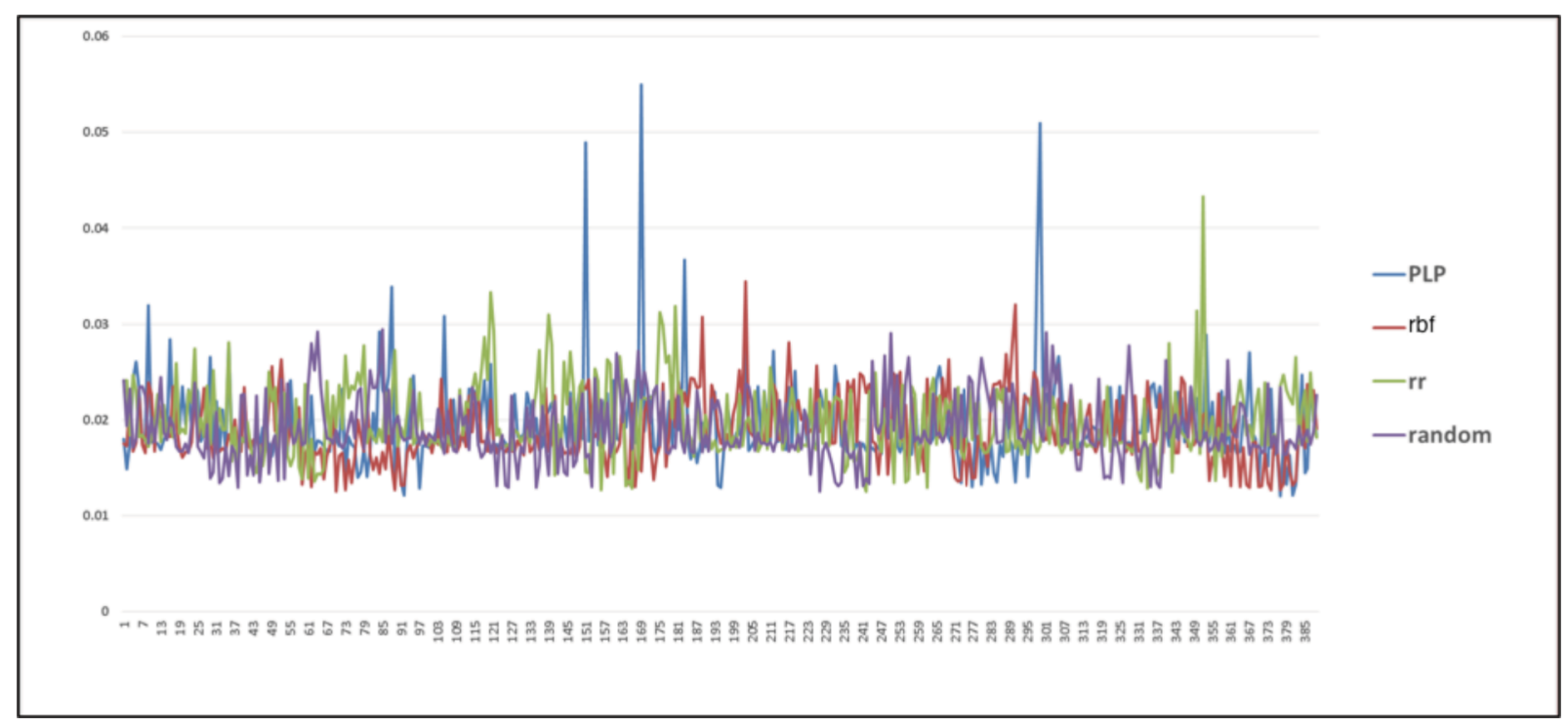

Figure 5. The loss of packets

The link delay between host and the real server is showing as Fig.4. We can see that all the load balancing methods have the similar performance and the average of link delay is below $0.02 \mathrm{~ms}$. The packet loss rate between hosts and real servers is showing as Fig.5. It's obvious that all the load balancing methods also have the similar performance and the highest rate of loss is below $1 \%$. Above that, we can conclude that the prediction strategy has a better performance on internet load balancing.

\section{Summary}

In this paper, we discuss the load balance on LVS and design an adaptive load balancing strategy. We implement the model with virtual machines for service request hosts and real machines for service reply servers. We created a new strategy that predicts the future traffic state and get the real status of each server in a certain time. The load server distribute the load depend on the future traffic and the weights of severs. The experiments results show that the new adaptive load balancing strategy has a better performance in load balance and has similar cost with other simple strategies. In the future, we will optimize the RBF's solving process and optimize the server weight coefficient. This work is supported by SKLSDE-2015ZX-07 project by State Key Laboratory of Software Development Environment of Beihang University and YCSJ-02-2015-05 project by Graduate Innovative Practice Fund of Beihang University.

\section{References}

1. Chen, Mike Y. \& Brewer, Eric. 2002. Active connection management in internet services. In
Network Operations and Management Symposium, 2002. NOMS 2002. 2002 IEEE/IFIP :265-280. IEEE.

2. Eunmi Choi, Yoojin Lim, and Dugki Min. Performace Comparison of Various Web Cluster Architectures [J]. Lecture Notes in Computer Science, May 2005, vol. 3398:617-624.

3. Ahmad I., Ghafoor A. and Mehrotra K. Performance Prediction of Distributed Load Balancing on Multicomputer Systems [J].ACM, 1991: 830-839.

4. Jain P. and Gupta D. An Algorithm for Dynamic Load Balancing in Distributed Systems with Multiple Supporting Nodes by Exploiting the Interrupt Service $[\mathrm{J}]$. Academy Publisher, 2009:232-236.

5. Information on http://www.linux-mag.com/2003-11/clusters_01.ht $\mathrm{ml}$

6. Hamdi M. and Lin C.K. Dynamic Load Balancing of Data Parallel Applications on a Distributed Network. In $9^{\text {th }}$ International Conference on Supercomputing, ACM, 1995: 170-179.

7. Information on http://www.linux-vs.org/

8. Canini, Marco. Kuznetsov, Petr. Levin, Dan. \& Schmid, Stefan. 2013. Software transactional networking: Concurrent and consistent policy composition. In Proceedings of the second ACM SIGCOMM workshop on Hot topics in software defined networking :1-6.ACM.

9. Mitchell, T.M. 2003. Machine learning. Beijing: China Machine Press.

10. Shi W, MacGregor M H, Gburzynski P. Load balancing for parallel forwarding. IEEE/ACM Transactions on Networking (TON), 2005, 13(4): 790-801 